Another protein with CNTF-like activity, growth promoting activity (GPA), is found in chick sciatic nerves ${ }^{19}$; unlike CNTF, GPA is secreted but it is not yet known whether it acts on mammalian motor neurons in vivo. Fibroblast growth factor- $5^{15}$, leukaemia-inhibitory factor ${ }^{1,20}$ and insulin-like growth factor ${ }^{4}$ also promote the survival of motor neurons in vitro, and seem to be made by muscle (see ref. 15), but they too have not been tested for their ability to rescue motor neurons in vivo. Whatever their roles turn out to be in the life of motor neurons, there is now no shortage of proteins that can be tested for their therapeutic value in treating degenerating motor neurons.

Anne W. Mudge is in the MRC Developmental Neurobiology Programme, Department of Biology, University College London, Gower Street, London WC1E 6BT, UK.

\title{
ESR on a single molecule
}

\section{Robert J. Silbey}

WITH condensed matter containing many billion billion molecules in every cubic centimetre, the newly acquired ability to pick out just one is remarkable. On pages 242 and 244 of this issue, respectively, J. Köhler et al. and J. Wrachtrup et al. describe experiments to obtain the electron magnetic resonance spectra of single molecules embedded in pentacene crystals. Because magnetic resonance spectra are sensitive measures of the shape of a molecule and of its interaction with surrounding molecules, this work opens up a new approach to molecular studies.

The experiments take advantage of the fact that in a solid at low temperature, the absorption spectra of individual guest molecules will have resonances at slightly different wavelengths owing to small differences in the intermolecular forces that each molecule experiences. This gives rise to a broadened spectral line made up of the sum of many single-molecule absorptions. In the wings of this broad line, the number of molecules contributing to the absorption decreases until far from the centre of the line, it is possible to measure (by fluorescence detection) the spectrum of a single molecule

The authors of the new work have previously found that some molecules have a spectrum that remains constant in time, while others have a spectrum that changes (spectral diffusion). The latter effect was attributed to time-dependent fluctuations in the surroundings of the absorbing molecule. Thus, both the static energy shift due to the intermolecular forces and the time dependence of these forces can be studied by this technique. Now these groups have added an important new tool - fluorescencedetected electron-spin-resonance (ESR) spectroscopy.

The researchers again study the singlemolecule spectrum by fluorescence detection, but this time they note that some of the molecules that are excited by light find themselves in a low-energy triplet state by the process of intersystem crossing. These molecules will stay in the triplet state long enough for the three energy levels of the triplet state to be probed with microwave radiation. These three states have different lifetimes, so by changing the state of the triplet state using microwave radiation (in other words, performing an ESR experiment while the molecule is in the triplet state), the fluorescence intensity is changed. Thus the ESR transitions in a single molecule are detected through the optical fluorescence.

The line shapes of the ESR transitions are of interest because they are a measure of the interactions between nuclear and electron spins (hyperfine interactions). Because the hydrogen nuclear spins relax slowly when the molecule is in the triplet state, one might expect that the line shape would be a signature of a single nuclear spin state, and therefore much narrower than the normal ESR line. Unfortunately, to detect the spectrum with sufficient signal to noise, the experiment has to be repeated on the same molecule many times. When the molecule is in a singlet state (ground or excited), the nuclear spins relax faster by interacting with the other nuclear spins in the crystal. Thus the average of many experiments is an average over all the different nuclear spin states, giving rise to a line shape identical to that seen in the normal many-molecule experiment.

So the experiments at present offer the tantalizing prospect of improved kinds of magnetic resonance experiments, such as ENDOR and optically detected nuclear spin polarization, which will eventually allow the study of the effects of the environment on the electronic structure of single molecules in crystals and in disordered or amorphous materials. Furthermore magnetic resonance spectroscopy may be ideal for elucidating the factors contributing to spectral diffusion.

Robert J. Silbey is in the Department of Chemistry, Massachusetts Institute of Technology, Cambridge, Massachusetts 02139, USA.
A right mess

THE messiest substance that Daedalus knows is a certain two-part silicone adhesive. Insidiously tacky, and with a low surface tension, it sticks copiously to anything it touches. It manages to get onto the handle of any spoon or spatula used to mix or transport it. Daedalus attributes this to the characteristic ability of thixotropic liquids to climb a rotated rod.

This masterpiece of messiness has been formulated quite unintentionally, without even any concept of messiness as a property of viscous liquids. So Daedalus reckons that a deliberate search should turn up some staggeringly messy self-spreading fluids. A team of tough-minded DREADCO chemists is now on the quest. Fluids of incredible exasperation are emerging, or rather escaping, from their laboratory. Handrails and carpets, tools and instruments round the company are developing a disquieting tackiness, and the chemists themselves are being shunned as a clique of untouchables. Daedalus is clearly onto something important.

"Hypergunge" (as it will be called) will probably sell on its sheer novelty at first, as bouncing putty and borate slime did before it. But serious uses should soon appear, such as the tracing of criminal contacts. A transparent and inconspicuous Hypergunge would be loaded with a sensitive fluorescer, or a halocarbon detectable in minute traces by an electronic 'sniffer'. A thief or burglar who stole an object protected with Hypergunge would not realize that he was now covered with it. But the resulting trail could easily be followed by the police; it might even spread via the criminal to his contacts and associates.

More humanely, Hypergunge could be used to spread insecticides among a reluctant population. The more gregarious and unhygienic that population, and therefore the more at risk from infestation, the more relentlessly it will spread all over them and their property. The insects, too, would find it getting lethally all over them and their hiding places. Similarly, a Hypergunge sheep-dip applied to a few animals would treat the whole flock, not to mention the farmworkers, their possessions, and their friends and relatives. In psychiatry, Hypergunge would provide perhaps the ultimate challenge to the obsessional handwasher. But novel containment and safety techniques will have to be developed. The consequences of a road accident to a tanker of Hypergunge do not bear thinking about. David Jones 